\title{
SUSTAINING MEMBERS
}

SePtember, 1934

COLLEGES, UNIVERSITIES, UNIVERSITY GROUPS, AND LEARNED SOCIETIES

Harvard University, Cambridge, Mass.

Institute for Advanced Study, Princeton, N.J.

Massachusetts Institute of Technology, Cambridge, Mass.

Mathematical Association of America, Oberlin, Ohio.

University of Chicago, Chicago, Ill.

University of Pennsylvania, Philadelphia, $\mathrm{Pa}$.

Yale University, New Haven, Conn.

LIFE INSURANCE COMPANIES AND INDUSTRIAL CONCERNS

Bell Telephone Laboratories, New York, N.Y.

Equitable Life Insurance Company of Iowa, Des Moines, Iowa.

John Hancock Mutual Life Insurance Company, Boston, Mass.

Metropolitan Life Insurance Company, New York, N.Y.

Prudential Insurance Company, Newark, N.J.

Western and Southern Life Insurance Company, Cincinnati, Ohio.

\section{INSTITUTIONAL CONTRIBUTING MEMBERS}

September, 1934

Northwestern University, Evanston, Ill.

Tulane University, New Orleans, La. 